Z. klin. Chem. u. klin. Biochem.

7. Jg., S. 179-180, März 1969

\title{
Nachwirkungen einmaliger Nebennierentindenstimulierung auf den Verlauf der Aldosteronausscheidung im Harn
}

\author{
Von G. WINkIER und V. Graef
}

Aus dem Pbysiol.-Chem. Institut (Direktor: Prof. Dr. Hj. Staudinger) der Universität Gießen, der Abteilung fïr Endokrinologie (Leiter: Prof. Dr. F. Babner) der Universität Heidelberg und der Abteilung fïr Endokrinologie und Stoffuvechsel (Leiter: Prof. Dr. E. F. Pfeiffer) der Universität Ulm

(Eingegangen am 2. Dezember 1968)

Herrn Prof. Dr. E. Tonutti żum 60. Geburtstag gewidmet

\begin{abstract}
Eine kräftige Stimulierung der Nebennierenrindenfunktion durch einen ACTH-Test wie auch bei einer körperlichen Belastung führt zu einem Anstieg der Ausscheidung von Aldosteron und Kalium im Harn und zu einem Rückgang der Natriumeliminierung. Im Anschluß daran kommt es zu einer teilweise verminderten Aldosteron- und Kalium-Ausscheidung, gefolgt von einem rebound-artigen Wiederanstieg. Er kann die während der Belastung gemessenen Werte überschreiten und im Fall des Aldosterons deutlich außerhalb des Normbereichs liegen. Dieses Phänomen sollte in der klinisch-chemischen Diagnostik bei der Beurteilung stark schwrankender Laboratoriums-Werte berücksichtigt werden.
\end{abstract}

\section{The effect of a single stimulation of the adrenal cortex on the course urinary aldosterone excretion}

Adrenal stimulation by ACTH or physical stress produces increased aldosterone and potassium excretion, coupled with sodium retention. This is followed by a decrease partly below control levels. A subsequent rebound-like rise partly exceeds the stimulation values. In order to avoid misinterpretations this should be taken into consideration when an unusually elevated hormone excretion is recorded.

Das Gleichgewicht im Elektrolythaushalt wird vornehmlich über verschiedene Regulationsmechanismen der Aldosteronsekretion der Nebennierenrinde (NNR) garantiert (s. 1,2). Ihre Anregung durch das adrenocorticotrope Hormon des Hypophysenvorderlappens (ACTH) wurde zwar früh betont (3), später verschiedentlich bestritten (u. a. 4) und durch in vivo- und in vitro-Untersuchungen überwiegend bestätigt (s. 1, 2, $3,5,6,7$ u. a.).

Bereits vor Jahren konnte in tierexperimentellen (8) und später in klinischen (9) Untersuchungen gezeigt werden, daß eine einmalige Stimulation der NNR durch ACTH von einem nachfolgenden phasischen Verlauf der 17-OHCS und 11-OCS-Ausscheidung im Harn gefolgt sein kann, der mit einem sog. „ReboundPhänomen" abgeschlossen wird. Im folgenden soll über gleichartige Beobachtungen des Verlaufs der Aldosteron- und Elektrolyt-Ausscheidung im Harn berichtet werden.

\section{Methodik}

Die Bestimmung des Aldosterons wurde nach der Methode von Novotny und Staudinger (10), die der Harnelektrolyte Natrium und Kalium flammenphotometrisch vorgenommen. Die Ergebnisse bei sechs endokrinologisch unauffälligen Probanden (3q, 30) im Alter von 20-50 Jahren, die eine einmalige i. m. Injektion von ACTH (200 bzw. 400 I. E. Depot-ACTH bzw. synthetisches ACTH (II. bzw. III. Intern. Standard) erhalten hatten, sind in Tabelle 1 und im Text aufgeführt. Bei einer weiteren Versuchsperson wurde die Ausscheidung von Aldosteron und den Harnelektrolyten nach einer körperlichen Belastung verfolgt.

\section{Ergebnisse}

Wie aus der Tabelle 1 ersichtlich ist, kommt es im Anschluß an die ACTH-Verabreichung zu einem deutlichen Anstieg der Aldosteronausscheidung von 8,0 \pm $2,12 \mu \mathrm{g}$ am Kontrolltag auf $24,4 \pm 1,43 \mu \mathrm{g} / \mathrm{Tag}$. Die Stimulierbarkeit der NNR zur Aldosteronsekretion durch ACTH wird somit erneut bewiesen.

Die Ausscheidungswerte fallen sodann zunächst bis zum Normbereich ab, wobei am 5. Tag ein kleiner Gipfel erkennbar ist. Vom 8. Tag nach der ACTHInjektion an setzt ein steiler Wiederanstieg der Aldo-

Tab. 1

Mittelwerte, mittlerer Fehler des Mittelwertes ( \pm S. E. M.) und Korrelationskoeffizienten der Harnmenge, Natrium-, Kalium- und Aldosteronausscheidung bei insgesamt 6 Versuchspersonen nach einmalige ACTH-Verabreichung:

(-1) entspricht dem Tag vor der ACTH-Gabe, +1 entspricht der Ausscheidungsmenge am Tag nach der Injektion

\begin{tabular}{|c|c|c|c|c|}
\hline Tag & $\begin{array}{l}\text { Harnmenge } \\
\mathrm{m} l / \text { Tag }\end{array}$ & $\begin{array}{c}\text { Natrium } \\
\text { mVal } / l / \text { Tag }\end{array}$ & $\begin{array}{c}\text { Kalium } \\
\text { mVal } / l / \text { Tag }\end{array}$ & $\begin{array}{c}\text { Aldosteron } \\
\mu \mathrm{g} / \mathrm{Tag}\end{array}$ \\
\hline $\begin{array}{r}-1 \\
+1 \\
2 \\
3 \\
4 \\
5 \\
6 \\
7 \\
8 \\
9 \\
9 \\
10 \\
11 \\
12\end{array}$ & $\begin{array}{r}918 \pm 223 \\
982 \pm 118 \\
486 \pm 86 \\
1010 \pm 235 \\
1070 \pm 246 \\
970 \pm 240 \\
886 \pm 245 \\
722 \pm 181 \\
970 \pm 239 \\
970 \pm 248 \\
874 \pm 176 \\
940 \pm 214 \\
530 \pm 98\end{array}$ & $\begin{array}{r}177,0 \pm 24,9 \\
86,4 \pm 16,0 \\
40,5 \pm 8,6 \\
197,6 \pm 40,2 \\
180,0 \pm 44,5 \\
189,4 \pm 60,1 \\
158,4 \pm 20,9 \\
145,4 \pm 41,4 \\
123,6 \pm 36,3 \\
141,8 \pm 46,8 \\
136,8 \pm 36,0 \\
146,6 \pm 52,7 \\
97,5 \pm 27,4\end{array}$ & $\begin{array}{l}41,2 \pm 6,8 \\
66,5 \pm 6,9 \\
24,8 \pm 4,6 \\
23,9 \pm 5,8 \\
40,1 \pm 12,6 \\
44,4 \pm 9,3 \\
40,9 \pm 9,5 \\
39,8 \pm 8,0 \\
49,7 \pm 7,0 \\
54,4 \pm 5,7 \\
59,5 \pm 9,0 \\
59,9 \pm 10,0 \\
59,8 \pm 9,8\end{array}$ & $\begin{array}{r}8,0 \pm 2,1 \\
24,4 \pm 1,4 \\
8,0 * \pm 4,7 \\
9,3 \pm 4,7 \\
11,2 \pm 2,9 \\
13,5 \pm 2,4 \\
10,4 \pm 3,7 \\
8,0 \pm 3,6 \\
14,3 \pm 0,5 \\
20,4 \pm 4,9 \\
26,3 \pm 3,1 \\
32,5 \pm 0,8 \\
24,3 \pm 7,7\end{array}$ \\
\hline
\end{tabular}

* wurde nicht errechnet

Korrelationskoeffizienten: $r$

$\begin{array}{ll}\text { Aldosteron/Kalium } & 0,87 \\ \text { Aldosteron/Natrium } & -0,34\end{array}$ 



Abb. 1

Verlauf der täglichen Ausscheidung von Aldosteron und Kalium im Harn bei einer gesunden männlichen Versuchsperson nach körper-
licher Belastung (Fußmarsch). Oben: Aldosteronausscheidung $\mu \mathrm{g} /$ 24 Stdn. Unten: Kaliumausscheidung mVal/24 Stdn.

steronausscheidung im Sinne eines "Rebound" ein, der mit über $30 \mu \mathrm{g} / \mathrm{Tag}$ auch die unter ACTH-Einwirkung gemessenen Werte weit überschreitet und außerhalb der mit dieser Methode (10) ermittelten Schwankungsbreite der Norm von 3,6-20,6 $\mu \mathrm{g} /$ Tag liegt. Wie die Ergebnisse der gleichzeitig verfolgten Elektrolytausscheidung im Harn (Tab. 1) ausweisen, geht erwartungsgemäß der Verlauf der täglichen Kaliumausscheidung den Aldosteronwerten parallel; der Korrelationskoeffizient beträgt 0,87 . Die Natrium-Elimination verhält sich nicht absolut spiegelbildlich (Korrelationskoeffi- zient-0,34); dabei ist zu bemerken, daß die Regulation der Natriumausscheidung neben dem AngiotensinMechanismus auch von einem sog. III.-Faktor abhängig sein soll (s. 11).

Der Spezifitätsbeweis der Stimulation der Aldosteronsekretion durch ACTH konnte in einem anderen Versuch erbracht werden. Ein Proband (26 Jahre $\delta^{7}$ ) erhielt $400 \mathrm{I}$. E. eines vollsynthetischen ACTH-Präparates (DW-75 „SANDOZ“) injiziert. Auch hier erfolgte ein Anstieg der Aldosteronausscheidung nach der ACTH-Gabe von $5 \mu \mathrm{g}$ auf $33 \mu \mathrm{g} / \mathrm{Tag}$, es folgte ein Abfall auf Werte zwischen 4-11 $\mu \mathrm{g} /$ Tag und ein Wiederanstieg von $22-32 \mu \mathrm{g} / \mathrm{Tag}$. Dieser stimmt mit dem Verhalten der Versuchsgruppe in Tabelle 1 überein.

$U_{m}$ zu prüfen, ob auch eine allgemeine Belastung mit gleichzeitiger endogener Erhöhung der ACTH-Ausschüttung zu einem vergleichbaren Verlauf der Aldosteronkurve führt, unterzog sich eine gesunde männliche Versuchsperson einem erheblichen Stress durch einen anstrengenden $\mathrm{Fußmarsch}$. Die Aldosteronausscheidung (Abb. 1) stieg von 5-12 $\mu \mathrm{g} / \mathrm{Tag}$ im Anschluß an die körperliche Belastung auf $28 \mu \mathrm{g} / \mathrm{Tag}$ an, fiel dann wieder auf Werte zwischen 3-14 $\mu \mathrm{g} / \mathrm{Tag}$ ab und erreichte 10 Tage nach dem Stress mit $26 \mu \mathrm{g} / \mathrm{Tag}$ erneut ein Maximum. Fast parallel dazu verlief auch die Kaliumausscheidung.

Unsere Ergebnisse zeigen somit in Übereinstimmung mit ähnlichen Befunden nach Belastung durch einen chirurgischen Eingriff (12), dạ eine einmalige, hinreichende NNR-Aktivierung zu merklichen Nachschwankungen der Aldosteronausscheidung sowie der mit ihr korrelierten Harnelektrolyte führt. Für die klinische Diagnostik erscheinen uns die mitgeteilten Befunde insofern bedeutungsvoll, àls im Hinblick auf die Schwierigkeit der Beurteilung derart schwankender Laborergebnisse nach zurückliegenden Belastungen gefahndet werden sollte.

Für die Úberlassung von Versuchsmengen Depot-ACTH sind wir der Firma Schering AG., Berlin, sehr zu Dank verpflichtet.

Die Firma Sandoz, Basel, stellte uns freundlicherweise Versuchsmengen von synthetischem ACTH (Präparat „DW-75“) zur Verfügung, wofür ihr herzliçhst gedankt sei.

\section{Literatur}

1. Gross, F., Acta endocr., K'hvn. Suppl. 124, 41 (1967). - 2. Wolff, H. P., Acta endocr., K'hvn. Suppl. 124, 65 (1967). 3. Liddel, G. W., J. Cornfield, A. G. T. Casper und F. C. Bartier, J. Clin. Endocr. Springfield 15, 852 (1955). - 4. Raitri, S., A. Kowarski, R. E. Morris und C. J. Mrgeon, Bull. Johns Hopkins Hosp. 119, 407 (1966). - 5. Mullek, A. F., A. M. RroNDEL und E. L. MANNING, Lancet London 1959/II 1021. 6. Laragh, J. H. und W. G. KelLy, Advances in Metabolic Disorders, Vol. 1, S. 217. Academ. Press, New York und London (1964). - 7. Tuccr, J. R., E. A. Espiner, P. I. JAGger, G. L.
Pauk und D. P. Lauler, J. Clin. Endocr. Springfield 27, 568 (1967). - 8. HerrmanN, M., Naturwissenschaften 48, 76 (1961). 9. Winkler, G., M. Herranann und A. Khalir, 12. Sympos. Deutsche Gesellschaft für Endokrinologie, Wiesbaden 1966, Springer-Verlag, Berlin-Heidelberg-New York (1967). - 10. 10. Nowotny, E. und HJ. Staudinger, diese Z. 4, 203 (1966). 11. Dölle, W., Internist 9,323 (1968). - 12. Venning, E., J. R. McCorrtston, I. Dyrenfurth und J. C. Beck, Metabolism Baltimore 7, 293 (1958).

\author{
Dr. G. Winkler \\ c/o Bereichsbibliothek des Zentrums für Innere Medizin \\ der Universität Ulm \\ 7900 Ulm (Donau) \\ Steinhövelstraße 9
}

